\title{
Parameter free induction and reflection
}

\author{
Lev D. Beklemishev* \\ Steklov Mathematical Institute \\ Vavilova 42, 117966 Moscow, Russia \\ e-mail: lev@bekl.mian.su
}

November 21, 1996

\begin{abstract}
We give a precise characterization of parameter free $\Sigma_{n}$ and $\Pi_{n}$ induction schemata, $I \Sigma_{n}^{-}$and $I \Pi_{n}^{-}$, in terms of reflection principles. This allows us to show that $I \Pi_{n+1}^{-}$is conservative over $I \Sigma_{n}^{-}$w.r.t. boolean combinations of $\Sigma_{n+1}$ sentences, for $n \geq 1$. In particular, we give a positive answer to a question by R. Kaye, whether the provably recursive functions of $I \Pi_{2}^{-}$are exactly the primitive recursive ones. We also obtain sharp results on the strength of bounded number of instances of parameter free induction in terms of iterated reflection.
\end{abstract}

\section{Introduction}

In this paper we shall deal with arithmetical theories containing Kalmar elementary arithmetic $E A$ or, equivalently, $I \Delta_{0}+\operatorname{Exp} . \Sigma_{n}$ and $\Pi_{n}$ formulas are prenex formulas obtained from the bounded ones by $n$ alternating blocks of similar quantifiers, starting from ' $\exists$ ' and ' $\forall$ ', respectively. $\mathcal{B}\left(\Sigma_{n}\right)$ denotes the class of boolean combinations of $\Sigma_{n}$ formulas. $\Sigma_{n}^{s t}$ and $\Pi_{n}^{s t}$ denote the classes of $\Sigma_{n}$ and $\Pi_{n}$ sentences.

Parameter free induction schemata have been introduced and investigated by Kaye, Paris, and Dimitracopoulos [11], Adamowicz and Bigorajska [1], Ratajczyk [13], Kaye [10], and others. $I \Sigma_{n}^{-}$is the theory axiomatized over $E A$ by the schema of induction

$$
A(0) \wedge \forall x(A(x) \rightarrow A(x+1)) \rightarrow \forall x A(x),
$$

for $\Sigma_{n}$ formulas $A(x)$ containing no other free variables but $x$, and $I \Pi_{n}^{-}$is similarly defined. $^{1}$

It is known that the schemata $I \Sigma_{n}^{-}$and $I \Pi_{n}^{-}$show a very different behaviour from their parametric counterparts $I \Sigma_{n}$ and $I \Pi_{n}$. In particular, for $n \geq 1, I \Sigma_{n}^{-}$and $I \Pi_{n}^{-}$are not finitely axiomatizable, and $I \Sigma_{n}^{-}$is strictly stronger than $I \Pi_{n}^{-}$(in fact, stronger than $\left.I \Sigma_{n-1}+I \Pi_{n}^{-}\right)$. Furthermore, it is known that $I \Sigma_{n}$ is a conservative extension of $I \Sigma_{n}^{-}$w.r.t. $\Sigma_{n+2}$ sentences, although $I \Sigma_{n}^{-}$itself only has a $\mathcal{B}\left(\Sigma_{n+1}\right)$ axiomatization [11].

In contrast, nontrivial conservation results for $I \Pi_{n}^{-}$, for $n>1$, seem to have been unknown. In particular, it was unknown, if the provably total recursive functions of $I \Pi_{2}^{-}$coincide with the primitive recursive ones (communicated by R. Kaye). The

\footnotetext{
* The research described in this publication was made possible in part by the Russian Foundation for Fundamental Research (project 93-011-16015).

${ }^{1}$ This definition differs from the one in [11] in that we work over $E A$, rather than over the weaker theories $I \Delta_{0}$ or $P A^{-}$. Since $I \Sigma_{1}^{-}$in the sense of [11] obviously contains $E A$, the two definitions are equivalent for $n \geq 1$ in $\Sigma$ case, and for $n \geq 2$ in $\Pi$ case.
} 
case of $I \Pi_{1}^{-}$(over $P A^{-}$) was essentially treated in [11], where the authors show that $\Pi_{2}$ consequences of that theory are contained in $E A$, cf also [6].

In this paper we prove that the provably total recursive functions of $I \Pi_{2}^{-}$are exactly the primitive recursive ones. Moreover, we show that $I \Pi_{n+1}^{-}$is conservative over $I \Sigma_{n}^{-}$w.r.t. boolean combinations of $\Sigma_{n+1}$ sentences $(n \geq 1)$. We also obtain sharp results on the strength of bounded number of instances of parameter free induction in terms of iterated reflection.

The proofs of these results are based on a characterization of parameter free induction schemata in terms of reflection principles and (generalizations of) the conservativity results for local reflection principles obtained in [3] using methods of provability logic. In our opinion, such a relationship presents an independent interest, especially because this seems to be the first occasion when local reflection principles naturally arise in the study of fragments of arithmetic.

We shall also essentially rely on the results from [4] characterizing the closures of arbitrary arithmetical theories extending $E A$ under $\Sigma_{n}$ and $\Pi_{n}$ induction rules. In fact, the results of this paper show that much of the unusual behaviour of parameter free induction schemata can be explained by their tight relationship with the theories axiomatized by induction rules.

\section{Preliminaries}

First, we establish some useful terminology and notation concerning rules in arithmetic (cf also [4]). We say that a rule is a set of instances, that is, expressions of the form

$$
\frac{A_{1}, \ldots, A_{n}}{B},
$$

where $A_{1}, \ldots, A_{n}$ and $B$ are formulas. Derivations using rules are defined in the standard way; $T+R$ denotes the closure of a theory $T$ under a rule $R$ and first order logic. $[T, R]$ denotes the closure of $T$ under unnested applications of $R$, that is, the theory axiomatized over $T$ by all formulas $B$ such that, for some formulas $A_{1}, \ldots, A_{n}$ derivable in $T, \frac{A_{1}, \ldots, A_{n}}{B}$ is an instance of $R . T \equiv U$ means that theories $T$ and $U$ are deductively equivalent, i.e., have the same set of theorems.

A rule $R_{1}$ is derivable from $R_{2}$ iff, for every theory $T$ containing $E A, T+$ $R_{1} \subseteq T+R_{2}$. A rule $R_{1}$ is reducible to $R_{2}$ iff, for every theory $T$ containing $E A$, $\left[T, R_{1}\right] \subseteq\left[T, R_{2}\right] . R_{1}$ and $R_{2}$ are congruent iff they are mutually reducible (denoted $R_{1} \cong R_{2}$ ). For a theory $U$ containing $E A$ we say that $R_{1}$ and $R_{2}$ are congruent modulo $U$, iff for every extension $T$ of $U,\left[T, R_{1}\right] \equiv\left[T, R_{2}\right]$.

Induction rule is defined as follows:

$$
\text { IR: } \quad \frac{A(0), \quad \forall x(A(x) \rightarrow A(x+1))}{\forall x A(x)} .
$$

Whenever we impose a restriction that $A(x)$ only ranges over a certain subclass $\Gamma$ of the class of arithmetical formulas, this rule is denoted $\Gamma$-IR. In general, we allow parameters to occur in $A$, however the following lemma holds (cf also [5]).

Lemma 2.1. $\Pi_{n}$-IR is reducible to parameter free $\Pi_{n}$-IR. $\Sigma_{n}$-IR is reducible to parameter free $\Sigma_{n}$-IR.

Proof. An application of IR for a formula $A(x, a)$ can obviously be reduced to the one for $\forall z A(x, z)$, and this accounts for the $\Pi_{n}$ case.

On the other hand, if $A(x, y, a)$ is $\Pi_{n-1}$, then an application of $\Sigma_{n}$-IR for the formula $\exists y A(x, y, a)$ is reducible, using the standard coding of sequences available in $E A$, to the one for $\exists y \forall i \leq x A\left((i)_{0},(y)_{i},(i)_{1}\right)$ (cf Remark 4.1 in [5]), q.e.d. 
Reflection principles, for a given r.e. theory $T$ containing $E A$, are defined as follows. The uniform reflection principle is the schema

$$
\operatorname{RFN}_{T}: \quad \forall x\left(\operatorname{Prov}_{T}(\ulcorner A(\dot{x})\urcorner) \rightarrow A(x)\right), \quad A(x) \text { a formula, }
$$

where $\operatorname{Prov}_{T}(\cdot)$ denotes a canonical provability predicate for $T$. The local reflection principle is the schema

$$
\operatorname{Rfn}_{T}: \quad \operatorname{Prov}_{T}(\ulcorner A\urcorner) \rightarrow A, \quad A \text { a sentence. }
$$

Partial reflection principles are obtained from the above schemata by imposing a restriction that $A$ belongs to one of the classes $\Gamma$ of the arithmetic hierarchy (denoted $\operatorname{Rfn}_{T}(\Gamma)$ and $\operatorname{RFN}_{T}(\Gamma)$, respectively). It is known that, due to the existence of partial truthdefinitions, the schema $\operatorname{RFN}_{T}\left(\Pi_{n}\right)$ is equivalent to a single $\Pi_{n}$ sentence over $E A$. In particular, $\operatorname{RFN}_{T}\left(\Pi_{1}\right)$ is equivalent to the consistency assertion $\operatorname{Con}_{T}$ for $T$. See $[14,12,3]$ for some basic information about reflection principles.

We shall also consider the following reflection rule:

$$
\operatorname{RR}\left(\Pi_{n}\right): \quad \frac{P}{\operatorname{RFN}_{E A+P}\left(\Pi_{n}\right)} .
$$

We let $\Pi_{m}-\operatorname{RR}\left(\Pi_{n}\right)$ denote the above rule with the restriction that $P$ is a $\Pi_{m}$ sentence. Main results (Theorems 1,2 and 3) of [4] can then be reformulated as follows.

Proposition 2.1. $\quad$ 1. $\Pi_{n}-\mathrm{IR} \cong \Pi_{n+1}-\mathrm{RR}\left(\Pi_{n}\right)$, for $n>1$;

2. $\Pi_{1}-\mathrm{IR} \cong \Pi_{2}-\mathrm{RR}\left(\Pi_{1}\right)\left(\bmod I \Delta_{0}+\right.$ Supexp$)$.

Proposition 2.2. 1. $\Sigma_{1}-\mathrm{IR} \cong \Pi_{2}-\mathrm{RR}\left(\Pi_{2}\right)$;

2. $\Sigma_{n}-\mathrm{IR} \cong \Pi_{n+1}-\mathrm{RR}\left(\Pi_{n+1}\right)\left(\bmod I \Sigma_{n-1}\right)$, for $n>1$.

Since $\left[E A, \Sigma_{n}\right.$-IR] contains $I \Sigma_{n-1}$, Statement 2 implies that the rules $\Pi_{n+1}-\operatorname{RR}\left(\Pi_{n+1}\right)$ and $\Sigma_{n}$-IR are interderivable, for all $n \geq 1$.

\section{Characterizing $I \Sigma_{n}^{-}$and $I \Pi_{n}^{-}$by reflection prin- ciples}

Theorem 1. For $n \geq 1$, over $E A$,

1. $I \Sigma_{n}^{-} \equiv\left\{P \rightarrow \operatorname{RFN}_{E A+P}\left(\Pi_{n+1}\right) \mid P \in \Pi_{n+1}^{s t}\right\}$;

2. $I \Pi_{n+1}^{-} \equiv\left\{P \rightarrow \operatorname{RFN}_{E A+P}\left(\Pi_{n+1}\right) \mid P \in \Pi_{n+2}^{s t}\right\}$.

Proof. Both statements are proved similarly, respectively relying upon Propositions 2.2 and 2.1, so we shall only elaborate the proof of the first one. For the inclusion $(\subseteq)$ we have to derive

$$
A(0) \wedge \forall x(A(x) \rightarrow A(x+1)) \rightarrow \forall x A(x),
$$

for each $\Sigma_{n}$ formula $A(x)$ with the only free variable $x$. We let $P$ denote the $\Pi_{n+1}$ sentence (logically equivalent to) $A(0) \wedge \forall x(A(x) \rightarrow A(x+1)$ ). Then, by external induction on $n$ it is easy to see that, for each $n, E A+P \vdash A(\bar{n})$. This fact is formalizable in $E A$, therefore

$$
E A \vdash \forall x \operatorname{Prov}_{E A+P}(\ulcorner A(\dot{x})\urcorner) .
$$


Denoting by $T$ the theory axiomatized over $E A$ by all formulas

$$
Q \rightarrow \operatorname{RFN}_{E A+Q}\left(\Pi_{n+1}\right)
$$

such that $Q$ is a $\Pi_{n+1}$ sentence, we conclude that

$$
\begin{aligned}
T+P & \vdash \operatorname{RFN}_{E A+P}\left(\Pi_{n+1}\right) \\
& \vdash \forall x\left(\operatorname{Prov}_{E A+P}(\ulcorner A(\dot{x})\urcorner) \rightarrow A(x)\right) \\
& \vdash \forall x A(x), \quad \text { by }(1) .
\end{aligned}
$$

It follows that $T \vdash P \rightarrow \forall x A(x)$, as required.

For the inclusion $(\supseteq)$ we observe that for any $\Pi_{n+1}$ sentence $P$ the theory $I \Sigma_{n}^{-}+P$ contains $P+\Sigma_{n}$-IR by Lemma 2.1 , and hence

$$
I \Sigma_{n}^{-}+P \vdash \operatorname{RFN}_{E A+P}\left(\Pi_{n+1}\right),
$$

by Proposition 2.2. It follows that

$$
I \Sigma_{n}^{-} \vdash P \rightarrow \operatorname{RFN}_{E A+P}\left(\Pi_{n+1}\right),
$$

q.e.d.

Remark 3.1. Statement 2 of the above theorem also holds for $n=0$, with a similar proof, but only over $I \Delta_{0}+$ Supexp (cf Corollary 4.2 below).

\section{Relativized provability and reflection}

For $n \geq 1, \Pi_{n}(\mathbf{N})$ denotes the set of all true $\Pi_{n}$ sentences. True $\Pi_{n}(x)$ denotes a canonical truthdefinition for $\Pi_{n}$ sentences, that is, a $\Pi_{n}$ formula naturally defining the set of Gödel numbers of $\Pi_{n}(\mathbf{N})$ sentences in $E A$. True $\Pi_{n}(x)$ provably in $E A$ satisfies Tarski satisfaction conditions (cf [9]), and therefore, for every formula $A\left(x_{1}, \ldots, x_{n}\right) \in \Pi_{n}$,

$$
E A \vdash A\left(x_{1}, \ldots, x_{n}\right) \leftrightarrow \operatorname{True}_{\Pi_{n}}\left(\left\ulcorner A\left(\dot{x}_{1}, \ldots, \dot{x}_{n}\right)\right\urcorner\right) .
$$

Tarski's truth lemma $(*)$ is formalizable in $E A$, in particular,

$$
E A \vdash \forall s \in \Pi_{n}^{s t} \operatorname{Prov}_{E A}\left(s \dot{\leftrightarrow}\left\ulcorner\operatorname{True}_{\Pi_{n}}(\dot{s})\right\urcorner\right),
$$

where $\Pi_{n}^{s t}$ is a natural elementary definition of the set of Gödel numbers of $\Pi_{n}$ sentences in $E A$. We also assume w.l.o.g. that

$$
E A \vdash \forall x\left(\operatorname{True}_{\Pi_{n}}(x) \rightarrow x \in \Pi_{n}^{s t}\right) .
$$

Let $T$ be an r.e. theory containing $E A$. A provability predicate for the theory $T+\Pi_{n}(\mathbf{N})$ can be naturally defined, e.g., by the following $\Sigma_{n+1}$ formula:

$$
\operatorname{Prov}_{T}^{\Pi_{n}}(x):=\exists s\left(\operatorname{True}_{\Pi_{n}}(s) \wedge \operatorname{Prov}_{T}(s \rightarrow x)\right) .
$$

Lemma 4.1. 1. For each $\Sigma_{n+1}$ formula $A\left(x_{1}, \ldots, x_{n}\right)$,

$$
E A \vdash A\left(x_{1}, \ldots, x_{n}\right) \rightarrow \operatorname{Prov}_{T}^{\Pi_{n}}\left(\left\ulcorner A\left(\dot{x}_{1}, \ldots, \dot{x}_{n}\right)\right\urcorner\right) .
$$

2. $\operatorname{Prov}_{T}^{\Pi_{n}}(x)$ satisfies Löb's derivability conditions in $T$. 
Proof. Statement 1 follows from (*). Statement 2 follows from Statement 1, Tarski satisfaction conditions, and is essentially well-known ( $\mathrm{cf}[15])$, q.e.d.

We define $\operatorname{Con}_{T}^{\Pi_{n}}:=\neg \operatorname{Prov}_{T}^{\Pi_{n}}(\ulcorner 0=1\urcorner)$, and relativized reflection principles $\operatorname{RFN}_{T}^{\Pi_{n}}$ and $\operatorname{Rfn}_{T}^{\Pi_{n}}$ are similarly defined. For $n=0$ all these schemata coincide, by definition, with their nonrelativized counterparts.

Lemma 4.2. For all $n \geq 0, m \geq 1$, the following schemata are deductively equivalent over $E A$ :

1. $\operatorname{Con}_{T}^{\Pi_{n}} \equiv \operatorname{RFN}_{T}\left(\Pi_{n+1}\right)$;

2. $\operatorname{Rfn}_{T}^{\Pi_{n}}\left(\Sigma_{m}\right) \equiv\left\{P \rightarrow \operatorname{RFN}_{T+P}\left(\Pi_{n+1}\right) \mid P \in \Pi_{m}^{s t}\right\}$.

Proof. 1. Observe that, using (**),

$$
\begin{aligned}
E A \vdash \neg \operatorname{Prov}_{T}^{\Pi_{n}}(\ulcorner 0=1\urcorner) & \leftrightarrow \neg \exists s\left(\operatorname{True}_{\Pi_{n}}(s) \wedge \operatorname{Prov}_{T}(s \dot{\rightarrow}\ulcorner 0=1\urcorner)\right) \\
& \leftrightarrow \forall s\left(\operatorname{Prov}_{T}(\dot{\neg} s) \rightarrow \neg \operatorname{True}_{\Pi_{n}}(s)\right) \\
& \leftrightarrow \forall s\left(\operatorname{Prov}_{T}\left(\left\ulcorner\neg \operatorname{True}_{\Pi_{n}}(\dot{s})\right\urcorner\right) \rightarrow \neg \operatorname{True}_{\Pi_{n}}(s)\right) .
\end{aligned}
$$

The latter formula clearly follows from $\operatorname{RFN}_{T}\left(\Sigma_{n}\right)$, but it also implies $\operatorname{RFN}_{T}\left(\Sigma_{n}\right)$, and hence $\operatorname{RFN}_{T}\left(\Pi_{n+1}\right)$, by $(*)$.

2. By formalized Deduction theorem,

$$
E A \vdash \operatorname{Con}_{T+P}^{\Pi_{n}} \leftrightarrow \neg \operatorname{Prov}_{T}^{\Pi_{n}}(\ulcorner\neg P\urcorner) .
$$

Hence, over $E A$,

$$
\begin{aligned}
\operatorname{Rfn}_{T}^{\Pi_{n}}\left(\Sigma_{m}\right) & \equiv\left\{\operatorname{Prov}_{T}^{\Pi_{n}}(\ulcorner S\urcorner) \rightarrow S \mid S \in \Sigma_{m}^{s t}\right\} \\
& \equiv\left\{P \rightarrow \neg \operatorname{Prov}_{T}^{\Pi_{n}}(\ulcorner\neg P\urcorner) \mid P \in \Pi_{m}^{s t}\right\} \\
& \equiv\left\{P \rightarrow \operatorname{RFN}_{T+P}\left(\Pi_{n+1}\right) \mid P \in \Pi_{m}^{s t}\right\}, \quad \text { by (2) and Statement 1, }
\end{aligned}
$$

q.e.d.

From this lemma and Theorem 1 we immediately obtain the following corollary.

Corollary 4.1. For $n \geq 1$, the following schemata are deductively equivalent over $E A$ :

1. $I \Sigma_{n}^{-} \equiv \operatorname{Rfn}_{E A}^{\Pi_{n}}\left(\Sigma_{n+1}\right)$;

2. $I \Pi_{n+1}^{-} \equiv \operatorname{Rfn}_{E A}^{\Pi_{n}}\left(\Sigma_{n+2}\right)$.

Corollary 4.2. Over $I \Delta_{0}+$ Supexp,

$$
I \Pi_{1}^{-} \equiv \operatorname{Rfn}_{E A}\left(\Sigma_{2}\right) \equiv \operatorname{Rfn}_{I \Delta_{0}+\operatorname{Supexp}}\left(\Sigma_{2}\right) .
$$

Proof. This follows in essentially the same way from Lemma 4.2 and the proof of Theorem 1, where we rely upon Proposition 2.1 (2) rather than (1). Indeed, over $I \Delta_{0}+$ Supexp,

$$
\begin{aligned}
I \Pi_{1}^{-} & \equiv\left\{P \rightarrow \operatorname{RFN}_{E A+P}\left(\Pi_{1}\right) \mid P \in \Pi_{2}^{s t}\right\} \\
& \equiv\left\{P \rightarrow \operatorname{Con}_{E A+P} \mid P \in \Pi_{2}^{s t}\right\} \\
& \equiv\left\{\operatorname{Prov}_{E A}(\ulcorner S\urcorner) \rightarrow S \mid S \in \Sigma_{2}^{s t}\right\} .
\end{aligned}
$$

Since the formula expressing the totality of superexponentiation function is a $\Pi_{2}$ sentence, the schemata $\operatorname{Rfn}_{I \Delta_{0}+\operatorname{Supexp}}\left(\Sigma_{2}\right)$ and $\operatorname{Rfn}_{E A}\left(\Sigma_{2}\right)$ are also equivalent over $I \Delta_{0}+$ Supexp, q.e.d. 


\section{Conservation results}

The following theorem is the main result of this paper.

Theorem 2. For any $n \geq 1, I \Pi_{n+1}^{-}$is conservative over $I \Sigma_{n}^{-}$w.r.t. $\mathcal{B}\left(\Sigma_{n+1}\right)$ sentences.

Proof. The result follows from Corollary 4.1 and the following relativized version of Theorem 1 of [3].

Theorem 3. For any r.e. theory $T$ containing $E A$ and any $n \geq 0, T+\operatorname{Rfn}_{T}^{\Pi_{n}}$ is conservative over $T+\operatorname{Rfn}_{T}^{\Pi_{n}}\left(\Sigma_{n+1}\right)$ w.r.t. $\mathcal{B}\left(\Sigma_{n+1}\right)$ sentences.

Proof. The proof of this theorem makes use of a purely modal logical lemma concerning Gödel-Löb provability logic GL (cf e.g. [7, 15]). Recall that GL is formulated in the language of propositional calculus endowed by a unary modal operator $\square$. The expressions $\diamond \phi$ and $\square^{+} \phi$ are standard abbreviations for $\neg \square \neg \phi$ and $\phi \wedge \square \phi$, respectively. Axioms of GL are all instances of propositional tautologies in this language together with the following schemata:

L1. $\square(\phi \rightarrow \psi) \rightarrow(\square \phi \rightarrow \square \psi)$;

L2. $\square \phi \rightarrow \square \square \phi$;

L3. $\square(\square \phi \rightarrow \phi) \rightarrow \square \phi$.

Rules of GL are moduls ponens and $\phi \vdash \square \phi$ (necessitation).

By an arithmetical realization of the language of GL we mean any function $(\cdot)^{*}$ that maps propositional variables to arithmetical sentences. For a modal formula $\phi,(\phi)_{T}^{*}$ denotes the result of substitution for all the variables of $\phi$ the corresponding arithmetical sentences and of translation of $\square$ as the provability predicate $\operatorname{Prov}_{T}(\ulcorner\cdot\urcorner)$. Under this interpretation, axioms L1, L2 and the necessitation rule can be seen to directly correspond to the three Löb's derivability conditions, and axiom L3 is the formalization of Löb's theorem. It follows that, for each modal formula $\phi$, GL $\vdash \phi$ implies $T \vdash(\phi)_{T}^{*}$, for every realization $(\cdot)^{*}$ of the variables of $\phi$. The opposite implication, for the case of a $\Sigma_{1}$ sound theory $T$, is also valid; this is the content of the important arithmetical completeness theorem for GL due to Solovay $(\mathrm{cf}[7])$.

For us it will also be essential that GL is sound under the interpretation of $\square$ as a relativized provability predicate. For an arithmetical realization $(\cdot)^{*}$, we let $(\phi)_{T+\Pi_{n}(N)}^{*}$ denote the result of substitution for all the variables of $\phi$ the corresponding arithmetical sentences and of translation of $\square$ as $\operatorname{Prov}_{T}^{\Pi_{n}}(\ulcorner\urcorner$.$) . The$ following lemma is a corollary of Lemma 4.1 .

Lemma 5.1. If GL $\vdash \phi$, then $T \vdash(\phi)_{T+\Pi_{n}(N)}^{*}$, for every arithmetical realization $(\cdot)^{*}$ of the variables of $\phi$.

The opposite implication, that is, the arithmetical completeness of GL w.r.t. the relativized provability interpretation, due to G. Boolos, is also well-known (cf [15]). Yet, below we do not use this fact.

The following crucial lemma is a modification of a similar lemma in [3].

Lemma 5.2. Let modal formulas $Q_{i}$ be defined as follows:

$$
Q_{0}:=p, \quad Q_{i+1}:=Q_{i} \vee \square Q_{i},
$$

where $p$ is a propositional variable. Then, for any variables $p_{0}, \ldots, p_{m}$,

$$
\mathbf{G L} \vdash \square^{+}\left(\bigwedge_{i=0}^{m}\left(\square p_{i} \rightarrow p_{i}\right) \rightarrow p\right) \rightarrow\left(\bigwedge_{i=0}^{m}\left(\square Q_{i} \rightarrow Q_{i}\right) \rightarrow p\right) .
$$


Proof. Rather than exhibiting an explicit proof of the formula above, we shall argue semantically, using a standard Kripke model characterization of GL.

Recall that a Kripke model for $\mathbf{G L}$ is a triple $(W, R, \Vdash)$, where

1. $W$ is a finite nonempty set;

2. $R$ is an irreflexive partial order on $W$;

3 . $\Vdash$ is a forcing relation between elements (nodes) of $W$ and modal formulas such that

$$
\begin{aligned}
x \Vdash \neg \phi & \Longleftrightarrow x \nVdash \phi, \\
x \Vdash(\phi \rightarrow \psi) & \Longleftrightarrow(x \nVdash \phi \text { or } x \Vdash \psi), \\
x \Vdash \square \phi & \Longleftrightarrow \forall y \in W(x R y \Rightarrow y \Vdash \phi) .
\end{aligned}
$$

Theorem 4 on page 95 of [7] (originally proved by Segerberg) states that a modal formula is provable in GL, iff it is forced at every node of any Kripke model of the above kind. This provides a useful criterion for showing provability in GL.

Consider any Kripke model $(W, R, \Vdash)$ in which the conclusion $\left(\bigwedge_{i=0}^{m}\left(\square Q_{i} \rightarrow\right.\right.$ $\left.Q_{i}\right) \rightarrow p$ ) is false at a node $x \in W$. This means that $x \nVdash p$ and $x \Vdash \square Q_{i} \rightarrow Q_{i}$, for each $i \leq m$. An obvious induction on $i$ then shows that $x \nVdash Q_{i}$ for all $i \leq m+1$, in particular, $x \nVdash Q_{m+1}$.

Unwinding the definition of $Q_{i}$ we observe that in $W$ there is a sequence of nodes

$$
x=x_{m+1} R x_{m} R \ldots R x_{0}
$$

such that, for all $i \leq m+1, x_{i} \nVdash Q_{i}$. Since $R$ is irreflexive and transitive, all $x_{i}$ 's are pairwise distinct. Moreover, it is easy to see by induction on $i$ that, for all $i$,

$$
\text { GL } \vdash p \rightarrow Q_{i} .
$$

Hence, for each $i \leq m+1, x_{i} \nVdash p$.

Now we notice that each formula $\square p_{i} \rightarrow p_{i}$ can be false at no more than one node of the chain $x_{m+1}, \ldots, x_{0}$. Therefore, by Pigeon-hole Principle, there must exist a node $z$ among the $m+2$ nodes $x_{i}$ such that

$$
z \Vdash \bigwedge_{i=0}^{m}\left(\square p_{i} \rightarrow p_{i}\right) \wedge \neg p .
$$

In case $z$ coincides with $x=x_{m+1}$ we have

$$
x \nVdash \bigwedge_{i=0}^{m}\left(\square p_{i} \rightarrow p_{i}\right) \rightarrow p .
$$

In case $z=x_{i}$, for some $i \leq m$, we have $x R z$ by transitivity of $R$, and thus

$$
x \nVdash \square\left(\bigwedge_{i=0}^{m}\left(\square p_{i} \rightarrow p_{i}\right) \rightarrow p\right) .
$$

This shows that the formula in question is forced at every node of any Kripke model; hence it is provable in $\mathbf{G L}$, q.e.d.

Lemma 5.3. For any $n \geq 0$ and any theory $T$, the following schemata are deductively equivalent over $E A$ :

$$
\operatorname{Rfn}_{T}^{\Pi_{n}}\left(\Sigma_{n+1}\right) \equiv \operatorname{Rfn}_{T}^{\Pi_{n}}\left(\mathcal{B}\left(\Sigma_{n+1}\right)\right)
$$


Proof: As in [3], using Lemma 4.1, q.e.d.

Now we complete our proof of Theorems 2 and 3 . Assume $T+\operatorname{Rfn}_{T}^{\Pi_{n}} \vdash A$, where $A$ is a $\mathcal{B}\left(\Sigma_{n+1}\right)$ sentence. Then there are finitely many instances of relativized local reflection that imply $A$, that is, for some arithmetical sentences $A_{0}, \ldots, A_{m}$, we have

$$
T \vdash \bigwedge_{i=0}^{m}\left(\operatorname{Prov}_{T}^{\Pi_{n}}\left(\left\ulcorner A_{i}\right\urcorner\right) \rightarrow A_{i}\right) \rightarrow A .
$$

Since relativized provability predicates satisfy Löb's derivability conditions, we also obtain

$$
T \vdash \operatorname{Prov}_{T}^{\Pi_{n}}\left(\left\ulcorner\bigwedge_{i=0}^{m}\left(\operatorname{Prov}_{T}^{\Pi_{n}}\left(\left\ulcorner A_{i}\right\urcorner\right) \rightarrow A_{i}\right) \rightarrow A\right\urcorner\right) .
$$

Considering an arithmetical realization $(\cdot)^{*}$ that maps the variable $p$ to the sentence $A$ and $p_{i}$ to $A_{i}$, for each $i$, by Lemma 5.2 we conclude that

$$
T \vdash \bigwedge_{i=0}^{m}\left(\operatorname{Prov}_{T}^{\Pi_{n}}\left(\left\ulcorner B_{i}\right\urcorner\right) \rightarrow B_{i}\right) \rightarrow A,
$$

where $B_{i}$ denote the formulas $\left(Q_{i}\right)_{T+\Pi_{n}(N)}^{*}$. Now we observe that, if $A \in \mathcal{B}\left(\Sigma_{n+1}\right)$, then for all $i, B_{i} \in \mathcal{B}\left(\Sigma_{n+1}\right)$. Hence

$$
T+\operatorname{Rfn}_{T}^{\Pi_{n}}\left(\mathcal{B}\left(\Sigma_{n+1}\right)\right) \vdash A,
$$

which yields Theorem 3 by Lemma 5.3. Theorem 2 follows from Theorem 3 and the observation that the schema $\operatorname{Rfn}_{E A}^{\Pi_{n}}\left(\Sigma_{n+2}\right)$ corresponding to $I \Pi_{n+1}^{-}$is actually weaker than the full $\operatorname{Rfn}_{E A}^{\Pi_{n}}$, q.e.d.

It is obvious, e.g., since $I \Sigma_{1}^{-}$contains $E A+\Sigma_{1}$-IR, that all primitive recursive functions are provably total recursive in $I \Sigma_{1}^{-}$and $I \Pi_{2}^{-}$. Moreover, since $I \Sigma_{1}^{-}$is contained in $I \Sigma_{1}$, by a well-known result of Parsons, any provably total recursive function of $I \Sigma_{1}^{-}$is primitive recursive. The following corollary strengthens this result and gives a positive answer to a question by R. Kaye.

Theorem 4. Provably total recursive functions of $I \Pi_{2}^{-}$are exactly the primitive recursive ones.

Proof: follows from $\mathcal{B}\left(\Sigma_{2}\right)$ conservativity of $I \Pi_{2}^{-}$over $I \Sigma_{1}^{-}$, q.e.d.

Remark 5.1. Observe that $I \Sigma_{1}+I \Pi_{2}^{-}$(unlike each of these theories taken separately) has a wider class of provably total recursive functions than the primitive recursive ones. This follows, e.g., from the fact that

$$
I \Sigma_{1}+I \Pi_{2}^{-} \vdash \mathrm{RFN}_{I \Sigma_{1}}\left(\Pi_{2}\right)
$$

by Theorem 1 (since $I \Sigma_{1}$ is equivalent to a $\Pi_{3}$ sentence).

Remark 5.2. Perhaps somewhat more naturally, conservation results for relativized local reflection principles can be stated modally within a certain bimodal system GLB due to Japaridze, with the operators $\square$ and $\square$, that describes the joint behaviour of the usual and the relativized provability predicate (cf [7]). Using a suitable Kripke model characterization of GLB, one can semantically prove that

$$
\mathbf{G L B} \vdash \square\left(\bigwedge_{i=0}^{m}\left(\square p_{i} \rightarrow p_{i}\right) \rightarrow p\right) \rightarrow \square\left(\bigwedge_{i=0}^{m}\left(\square Q_{i} \rightarrow Q_{i}\right) \rightarrow p\right),
$$

where the formulas $Q_{i}$ are now understood w.r.t. the modality $\square$, and this yields Theorem 3 almost directly. 


\section{Axiomatization results}

The characterization of parameter free induction in terms of reflection principles (Theorem 1) actually reveals other interesting information about these schemata.

The following theorem, which is a corollary of the relativized version of another conservation result for local reflection principles (due, essentially, to Goryachev [8]), gives a characterization of $\Pi_{n+1}$ consequences of $I \Sigma_{n}^{-}$and $I \Pi_{n+1}^{-}$. For the case of $I \Sigma_{n}^{-}$a related charcterization of provably total recursive functions is given in $[1,13]$. On the other hand, the paper [11] also contains a related conservation result for $I \Pi_{1}^{-}$w.r.t. $\Pi_{1}$ sentences $\left(I \Pi_{1}^{-}\right.$is formulated over $\left.P A^{-}\right)$.

Let $T$ be an r.e. theory containing $E A$. For a fixed $n \geq 1$, we define a sequence of theories $(T)_{i}^{n}$ as follows:

$$
(T)_{0}^{n}:=T ; \quad(T)_{i+1}^{n}:=(T)_{i}^{n}+\operatorname{RFN}_{(T)_{i}^{n}}\left(\Pi_{n}\right) ; \quad(T)_{\omega}^{n}:=\bigcup_{i \geq 0}(T)_{i}^{n} .
$$

Theorem 5. 1. Let $U_{m}$ be a theory axiomatized over $E A$ by arbitrary $m$ instances of $I \Pi_{n+1}^{-}, n \geq 1$. Then $U_{m}$ is $\Pi_{n+1}$ conservative over $(E A)_{m}^{n+1}$.

2. There exist particular $m$ instances $U_{m}$ of $I \Pi_{n+1}^{-}$(in fact, of $I \Sigma_{n}^{-}$) such that $U_{m}$ contains $(E A)_{m}^{n+1}$.

Proof. The proof relies on the fact that our characterization of parameter free induction schemata in terms of reflection principles respects the number of instances of these schemata.

Lemma 6.1. For every instance $B$ of $I \Pi_{n+1}^{-}$there is a $\Pi_{n+2}$ sentence $P$ such that $P \rightarrow \operatorname{RFN}_{E A+P}\left(\Pi_{n+1}\right)$ implies $B$ over $E A$. Vice versa, for every such $P$ there is an instance $B$ of $I \Pi_{n+1}^{-}$such that $E A+B$ proves $P \rightarrow \operatorname{RFN}_{E A+P}\left(\Pi_{n+1}\right)$.

Proof: follows directly from our proof of Theorem 1. For the 'vice versa' part we employ Proposition 2.1 (1) stating that

$$
\left[E A+P, \Pi_{n+1}-\mathrm{IR}\right] \vdash \mathrm{RFN}_{E A+P}\left(\Pi_{n+1}\right),
$$

and the fact that any finite number of unnested applications of $\Pi_{n+1}$-IR can be obviously merged into a single one, q.e.d.

Remark 6.1. A similar statement holds for $I \Sigma_{n}^{-}$, but only over $I \Sigma_{n-1}$. In general one seems to need $m+1$ instances of $I \Sigma_{n}^{-}$in order to derive $m$ instances of the corresponding reflection schema (the first one is used to derive $I \Sigma_{n-1}$ ).

Let $\perp$ denote the boolean constant 'falsum'.

Lemma 6.2. GL $\vdash \square^{+} \neg \bigwedge_{i=0}^{m}\left(\square p_{i} \rightarrow p_{i}\right) \rightarrow \square^{m+1} \perp$.

Proof. By Lemma 5.2 we have

$$
\mathbf{G L} \vdash \square^{+}\left(\bigwedge_{i=0}^{m}\left(\square p_{i} \rightarrow p_{i}\right) \rightarrow p\right) \rightarrow\left(\bigwedge_{i=0}^{m}\left(\square Q_{i} \rightarrow Q_{i}\right) \rightarrow p\right) .
$$

Then, substituting in the above formula $\perp$ for $p$, observe that

$$
\mathbf{G L} \vdash Q_{i}(p / \perp) \leftrightarrow \square^{i} \perp,
$$

and therefore

$$
\mathbf{G L} \vdash \bigwedge_{i=0}^{m}\left(\square Q_{i}(p / \perp) \rightarrow Q_{i}(p / \perp)\right) \leftrightarrow \neg \square^{m+1} \perp,
$$


q.e.d.

Now we complete the proof of Theorem 5. For the first part, Lemma 6.1 implies that $U_{m}$ is contained in a theory axiomatized by $m$ instances of relativized local reflection, say $\operatorname{Prov}_{E A}^{\Pi_{n}}\left(\left\ulcorner A_{i}\right\urcorner\right) \rightarrow A_{i}$, for $i<m$. Let $A$ be a $\Pi_{n+1}$ sentence such that $E A+U_{m} \vdash A$. Then we have

$$
E A \vdash \neg A \rightarrow \neg \bigwedge_{i=0}^{m-1}\left(\operatorname{Prov}_{E A}^{\Pi_{n}}\left(\left\ulcorner A_{i}\right\urcorner\right) \rightarrow A_{i}\right)
$$

and, by Löb's derivability conditions,

$$
E A \vdash \operatorname{Prov}_{E A}^{\Pi_{n}}(\ulcorner\neg A\urcorner) \rightarrow \operatorname{Prov}_{E A}^{\Pi_{n}}\left(\left\ulcorner\neg \bigwedge_{i=0}^{m-1}\left(\operatorname{Prov}_{E A}^{\Pi_{n}}\left(\left\ulcorner A_{i}\right\urcorner\right) \rightarrow A_{i}\right)\right\urcorner\right) .
$$

By Lemma 6.2 we then obtain

$$
\begin{aligned}
E A \vdash\left(\neg \square^{m} \perp\right)_{E A+\Pi_{n}(N)}^{*} & \rightarrow\left(A \vee \neg \operatorname{Prov}_{E A}^{\Pi_{n}}(\ulcorner\neg A\urcorner)\right) \\
& \rightarrow A,
\end{aligned}
$$

by Lemma 4.1 (1). Statement 1 of Lemma 4.2 implies that, for all $i$,

$$
(T)_{i}^{n+1} \vdash\left(\neg \square^{i} \perp\right)_{E A+\Pi_{n}(N)}^{*},
$$

therefore $(E A)_{m}^{n+1} \vdash A$, which shows the first claim of Theorem 5 .

For the second claim, we use a result from [4] stating that $m$ times iterated uniform $\Pi_{n+1}$ reflection principle over $E A$ is derivable by $m$ nested applications of $\Pi_{n+1}$-IR, and even $\Sigma_{n}$-IR, that is,

$$
(E A)_{m}^{n+1} \subseteq\left[\ldots\left[E A, \Sigma_{n}-\mathrm{IR}\right], \ldots, \Sigma_{n}-\mathrm{IR}\right] \quad(m \text { times }) .
$$

(For the case of $\Pi_{n+1}$-IR this statement obviously follows from Proposition 2.1. For the case of $\Sigma_{n}$-IR we need an additional observation that $\left[E A, \Sigma_{n}\right.$-IR $] \equiv I \Sigma_{n-1}$.) Hence, particular $m$ instances $U_{m}$ of $I \Sigma_{n}^{-}$can be found such that $E A+U_{m}$ contains $(E A)_{m}^{n+1}$, q.e.d.

Remark 6.2. The first statement of Theorem 5 is also valid for $n=0$, but over $I \Delta_{0}+$ Supexp rather than $E A$. A proof is similar, using Corollary 4.2. For $E A$ a similar characterization can be obtained using bounded cut-rank provability a là Wilkie and Paris [16], cf also [4].

Corollary 6.1. For $n \geq 1, I \Pi_{n+1}^{-}$and $I \Sigma_{n}^{-}$are $\Pi_{n+1}$ conservative extensions of $(E A)_{\omega}^{n+1}$.

The following corollary was proved model-theoretically in [11].

Corollary 6.2. For $n \geq 1$, neither $I \Sigma_{n}^{-}$, nor $I \Pi_{n+1}^{-}$is finitely axiomatizable.

This corollary can be strengthened by using the following two generalizations of Theorem 5 . They both are proved in essentially the same way as Theorem 5 , so we omit their proofs.

Theorem 6. Let $T$ be an extension of $E A$ by finitely many $\Pi_{n+2}$ sentences, $n \geq 1$. Then

1. For any $m$ instances $U_{m}$ of of $I \Pi_{n+1}^{-}, T+U_{m}$ is $\Pi_{n+1}$ conservative over $(T)_{m}^{n+1}$. 
2. There exist particular $m$ instances $U_{m}$ of $I \Pi_{n+1}^{-}$such that $T+U_{m}$ contains $(T)_{m}^{n+1}$.

Theorem 7. Let $T$ be an extension of $E A$ by finitely many $\Pi_{n+1}$ sentences, $n \geq 1$. Then

1. For any $m$ instances $U_{m}$ of of $I \Sigma_{n}^{-}, T+U_{m}$ is $\Pi_{n+1}$ conservative over $(T)_{m}^{n+1}$.

2. There exist particular $m+1$ instances $U_{m+1}$ of $I \Sigma_{n}^{-}$such that $T+U_{m+1}$ contains $(T)_{m}^{n+1}$.

Corollary 6.3. No consistent extension of $I \Pi_{n+1}^{-}$by $\Pi_{n+2}$ sentences is finitely axiomatizable.

Proof. Suppose, on the contrary, that there is such an extension. We may assume w.l.o.g. that it has the form $T+U_{m}$, for some $m$ instances $U_{m}$ of $I \Pi_{n+1}^{-}$, where $T$ is a finite $\Pi_{n+2}$ axiomatized extension of $E A$. Then, by Theorem $6, \Pi_{n+1}$ consequences of $T+U_{m}$ coincide with those of $(T)_{m}^{n+1}$, yet

$$
T+I \Pi_{n+1}^{-} \vdash \operatorname{RFN}_{(T)_{m}^{n+1}}\left(\Pi_{n+1}\right) .
$$

The latter formula is $\Pi_{n+1}$ and unprovable in $(T)_{m}^{n+1}$, q.e.d.

Corollary 6.4. No consistent extension of $I \Sigma_{n}^{-}$by $\Pi_{n+1}$ and $\Sigma_{n+1}$ sentences is finitely axiomatizable.

Proof. Suppose, on the contrary, that there is such an extension. We may assume w.l.o.g. that it has the form $T+U_{m}+S$, for some $m$ instances $U_{m}$ of $I \Sigma_{n}^{-}, S$ a $\Sigma_{n+1}$ sentence, and $T$ a finite $\Pi_{n+1}$ axiomatized extension of $E A$. Then, by Theorem 7 , $\Pi_{n+1}$ consequences of $T+U_{m}$ are contained in those of $(T)_{m}^{n+1}$, in particular, if $T+U_{m} \vdash \neg S$, then $(T)_{m}^{n+1} \vdash \neg S$. Moreover, this fact is formalizable in $T$ (to see this it is convenient to apply Lemma 5.2 once again), so that $\operatorname{Con}_{(T)_{m}^{n+1}+S}$ implies $\operatorname{Con}_{T+U_{m}+S}$ and $\operatorname{Con}_{T+I \Sigma_{n}^{-}+S}$, by our choice of $U_{m}$. Yet, by Theorem 7 ,

$$
T+I \Sigma_{n}^{-} \vdash \operatorname{RFN}_{(T)_{m}^{n+1}}\left(\Pi_{n+1}\right),
$$

therefore

$$
T+I \Sigma_{n}^{-}+S \vdash \operatorname{Con}_{(T)_{m}^{n+1}+S},
$$

contradicting Gödel's second incompleteness theorem, q.e.d.

\section{References}

[1] Z. Adamovicz and T. Bigorajska. Functions provably total in $I^{-} \Sigma_{1}$. Fundamenta mathematicae, 132:189-194, 1989.

[2] Z. Adamovicz and R. Kossak. A note on $B \Sigma_{n}$ and an intermediate induction schema. Zeitschrift f. math. Logik und Grundlagen d. Math., 34:261-264, 1988.

[3] L.D. Beklemishev. Notes on local reflection principles. Logic Group Preprint Series 133, University of Utrecht, 1995.

[4] L.D. Beklemishev. Induction rules, reflection principles, and provably recursive functions. Logic Group Preprint Series 168, University of Utrecht, 1996.

[5] L.D. Beklemishev. A proof-theoretic analysis of collection. Submitted to Archive for Mathematical Logic, 1996. 
[6] T. Bigorajska. On $\Sigma_{1}$-definable functions provably total in $I \Pi_{1}^{-}$. Mathematical Logic Quarterly, 41:135-137, 1995.

[7] G. Boolos. The Logic of Provability. Cambridge University Press, Cambridge, 1993.

[8] S. Goryachev. On interpretability of some extensions of arithmetic. Mat. Zametki, 40:561-572, 1986. In Russian.

[9] P. Hájek and P. Pudlák. Metamathematics of First Order Arithmetic. SpringerVerlag, Berlin, Heidelberg, New-York, 1993.

[10] R. Kaye. Parameter free universal induction. Zeitschrift f. math. Logik und Grundlagen d. Math., 35(5):443-456, 1989.

[11] R. Kaye, J. Paris, and C. Dimitracopoulos. On parameter free induction schemas. Journal of Symbolic Logic, 53(4):1082-1097, 1988.

[12] G. Kreisel and A. Lévy. Reflection principles and their use for establishing the complexity of axiomatic systems. Zeitschrift f. math. Logik und Grundlagen d. Math., 14:97-142, 1968.

[13] Z. Ratajczyk. Functions provably total in $I^{-} \Sigma_{n}$. Fundamenta Mathematicae, 133:81-95, 1989.

[14] C. Smoryński. The incompleteness theorems. In J. Barwise, editor, Handbook of Mathematical Logic, pages 821-865. North Holland, Amsterdam, 1977.

[15] C. Smoryński. Self-Reference and Modal Logic. Springer-Verlag, BerlinHeidelberg-New York, 1985.

[16] A. Wilkie and J. Paris. On the scheme of induction for bounded arithmetic formulas. Annals of Pure and Applied Logic, 35:261-302, 1987. 